\title{
Guiding coronary revascularization using PET stress myocardial perfusion imaging: The proof is in the pudding
}

\author{
Ajay V. Srivastava, MD, FACC, and Karthik Ananthasubramaniam, MD \\ FRCP(Glas)FACC FASE FASNC ${ }^{b}$
}

a Scripps Clinic Torrey Pines, La Jolla, CA

${ }^{\mathrm{b}}$ Heart and Vascular Institute, Henry Ford Hospital, Detroit, MI

Received Mar 13, 2016; accepted Mar 14, 2016

doi: 10.1007/s12350-016-0479-2

See related article, pp. 961 -974

Robust evidence over many years supports the widespread use of pharmacologic rubidium- 82 positron emission tomography (PET) myocardial perfusion imaging (MPI) for noninvasive detection of coronary artery disease (CAD) as well as for risk stratification of patients with known CAD. ${ }^{1,2}$ In early experimental studies, ${ }^{3}$ critical myocardial ischemia had been defined during acute coronary occlusion as flow reduction of $50 \%$. Gould et $\mathrm{al}^{4}$ established that the fraction of left ventricle myocardium rendered critically ischemic during acute coronary occlusion can be measured accurately and noninvasively using perfusion imaging with PET. Hachamovitch et $\mathrm{al}^{5}$ determined that patients with $>10 \%$ ischemic myocardium as evaluated by SPECT had lower death rates when subjected to early revascularization compared to medical therapy whereas lesser amounts of ischemia fared better with initial strategy of medical therapy. This concept appears to guide current considerations for medical therapy versus revascularization in clinical practice using both SPECT and PET. Estimation of ischemia by PET is further strengthened by assessing various parameters such as stress left ventricular ejection fraction and reserve, and

Reprint requests: Karthik Ananthasubramaniam, MD FRCP(Glas)FACC FASE FASNC, Heart and Vascular Institute, Henry Ford Hospital, 2799 West Grand Blvd, K-14, Detroit, MI 48202; kananth1@hfhs.org

J Nucl Cardiol 2017;24:975-9.

$1071-3581 / \$ 34.00$

Copyright (c) 2016 American Society of Nuclear Cardiology. presence of transient ischemic dilation of the left ventricle. ${ }^{5-7}$

Although visual estimation of perfusion defects with PET still focuses on relative perfusion, one of the major advantages of PET with its higher temporal and spatial resolution, robust attenuation correction and tracer kinetic modeling methods, is the ability to calculate myocardial blood flow (MBF) in $\mathrm{mL} \cdot \mathrm{min}^{-1} \cdot \mathrm{g}^{-1}$ of tissue. Thus, quantitative PET holds great promise in enhancing diagnostic accuracy of coronary disease and shedding light into areas where relative perfusion struggles, namely balanced ischemia, correct identification of three vessel disease, and microvascular disease.

Evaluating regional MBF with PET using N-13 ammonia and O-15 labeled water has been widely validated but requires an on-site cyclotron for their production. Being generator produced, rubidium- 82 is now the most widely used radionuclide for PET perfusion imaging. There are inherent limitations with MBF quantification using rubidium-82 such as nonlinear extraction fraction and higher positron range leading to greater image degradation. However, several studies ${ }^{8}$ have now been published validating rubidium- 82 using a single compartmental model as reliable method for MBF quantitation. Furthermore, several studies have also now demonstrated the incremental prognostic value of PET derived myocardial flow reserve (MFR) over clinical factors, perfusion defect size, and severity in patients with known or suspected CAD. ${ }^{9-12}$ A quest for further refining the prognostic value of PET MPI has led to significant developments in the past decade in the area of assessment of rest and vasodilator stress MBF and thus calculation of regional MFR or coronary blood flow reserve (CFR) by PET MPI. ${ }^{7,9,10}$ For the purpose of this editorial and to be consistent with the study reviewed, ${ }^{13}$ we will use the term CFR although both CFR and MFR are used interchangeably in the literature. 
Measurement of rest and peak stress MBF and CFR correlate inversely to coronary artery stenosis severity. From review of published studies on CFR, it is increasingly evident that patients with reduced CFR experience higher cardiac mortality even after accounting for differences in clinical factors and extent and severity of perfusion defects, ${ }^{14}$ and hence, MBF assessment is entering into routine clinical practice. It is important to recall that CFR assessed by vasodilator stress is predominantly a measure of endothelium-independent coronary flow abnormalities and to a lesser extent endothelium-dependent flow abnormalities caused by coronary artery stenosis. Hence, CFR is more a global measure of vasculature and evaluation and interpretation of hyperemic MBF, and CFR with PET MPI needs to be set in the clinical context with coronary anatomy and concomitant cardiovascular risk factors. This has been postulated as the reason why specific patient cohorts show significant improvements in CFR despite no mechanical revascularization but with aggressive dietary and lifestyle modifications, such as low fat diets and tobacco abstinence. ${ }^{15,16}$ This may also explain why hyperemic MBF has proved to be more accurate than CFR in detecting flow-limiting stenosis on both a per patient analysis and a per vessel analysis.

While there is a significant amount of evidence supporting the prognostic utility of pharmacologic rubidium-82 PET MPI, there is a paucity of prospective clinical trials utilizing PET MPI data to drive patient management, such as to guide coronary angiography or revascularization decisions. Based on published studies, ${ }^{17-19}$ invasive coronary angiography is deemed appropriate for patients with high-risk findings on PET MPI such as significant ischemic perfusion defects involving $>10 \%$ ischemic myocardium and other highrisk markers, such as transient ischemic dilation, stressinduced left ventricle dysfunction, or significant electrocardiographic changes during stress testing. While the COURAGE (Clinical Outcomes Utilizing Revascularization and Aggressive Drug Evaluation) trial showed us that percutaneous coronary intervention (PCI) based on angiographic stenosis severity did not reduce coronary events compared to guideline-directed medical treatment ${ }^{20}$ trials with invasive fractional flow reserve (FFR) assessment (FAME Trial-Fractional Flow Reserve Versus Angiography for Multivessel Evaluation) suggest that FFR measurement can be a useful tool in the revascularization decision-making process and lead to better outcomes. ${ }^{21}$ While the COURAGE and FAME trials addressed different aspects of stable CAD, both of these trials highlight the relevance of physiologic impact of stenosis compared to anatomic stenosis in revascularization decision making. Furthermore studies ${ }^{4,22}$ have elegantly investigated this anatomic versus physiologic discordance between FFR and CFR and highlighted the unique role of PET in the revascularization decisionmaking process. It is well known that FFR measures stenosis related decline in distal coronary pressure during maximum hyperemia. The normal coronary vessel exerts minimal resistance to flow both during rest and hyperemia, but in the presence of a focal coronary artery stenosis, the distal coronary pressure declines during hyperemia as a function of the stenosis severity and stenosis with FFRs $<0.75$ or $<0.80$ have been shown to induce ischemia and are defined as functionally significant. This is a vital difference compared to CFR, which measures the ratio of maximal hyperemic to resting flows for a given artery with or without stenosis or diffuse narrowing. Thus, FFR and CFR may represent different aspects of the disease process. This difference is most apparent in patients with diffuse CAD, leading to significantly low CFR but minimal change in FFR due to the absence of focal stenosis and hence preservation of pressure-derived flow reserve. The discordance between FFR and CFR may be as high as $40 \%$, as shown in some studies. ${ }^{22,23}$ These scenarios happen when focal stenosis is accompanied by preserved global MBF and CFR and vice versa when diffuse microvascular disease and abnormal CFR is accompanied by minimal abnormalities in FFR. ${ }^{22-24}$

In this issue of the journal, Bober et $\mathrm{al}^{13}$ sought to investigate changes in PET determined MBF, specifically stress MBF (sMBF), in a small group $(n=19)$ of patients undergoing pharmacologic rubidium-82 PET MPI pre (PET 1)- and post (PET 2)-percutaneous coronary intervention (PCI). The most common indications for the PET scans was chest pain and the population tested was intermediate to high-risk group overall. The main focus of their study was to assess if revascularization based on the presence of PET reversible perfusion defects is associated in improvement of regional MBF pertaining to those segments. Toward this purpose, they classified segments into four categories based on the presence or absence of PET perfusion defects and if PCI was performed in those segments or not (Table 1 of their paper). Of note, a 17-segment model was not used to classify these segments, but rather a 4-quadrant program was used to classify defects. For reasons stated above emphasizing the differences between CFR and FFR assessment, the authors focused specifically on hyperemic or stress MBF (sMBF) and not CFR. Prior investigators have also suggested the role of "hyperemic MBF assessment" as opposed to "CFR' as potentially a better parameter to guide patient management. ${ }^{23-25}$

Following matched revascularization (presence of PET reversible defect and PCI of that segment), post-revascularization $\mathrm{sMBF}$ increased by $0.6 \pm 0.7 \mathrm{~mL} \cdot \mathrm{min}^{-1} \cdot \mathrm{g}^{-1}$ 
$(1.2 \pm 0.4$ vs $1.7 \pm 0.8 ; P<.001)$ and CFR increased by $0.5 \pm 0.6(1.3 \pm 0.4$ vs $1.8 \pm 0.7 ; P=.001)$. The average stress induced perfusion defect size decreased from $20 \%$ to $8 \%$ of the entire left ventricle myocardium $(P<.001)$ and average $\mathrm{SMBF}$ and CFR within the defects improved from 0.59 to $0.96 \mathrm{~mL} \cdot \mathrm{min}^{-1} \cdot \mathrm{g}^{-1}(P=.05)$ and from 0.77 to 1.13 $(P=.02)$, respectively. In the unmatched revascularization group (no defect but revascularization was performed; $\mathrm{n}=14$ ), neither stress MBF nor CFR showed a significant change $\left(1.7 \pm 0.3\right.$ vs $1.5 \pm 0.4 \mathrm{~mL} \cdot \mathrm{min}^{-1} \cdot \mathrm{g}^{-1}, P=.16$; and $1.4 \pm 0.2$ vs $1.5 \pm 0.3, P=.11$; respectively). There was no significant change in the average stress-induced perfusion defect size either (4\% vs $10 \%$ of the myocardium, $P=.41)$. There also was no significant change in the average minimal sMBF or CFR within the defects (1.18 vs $0.84 \mathrm{~mL} \cdot \mathrm{min}^{-1} \cdot \mathrm{g}^{-1}, P=.11$; and 0.98 vs $0.97, P=.93$; respectively). Importantly there was no increase in SMBF in unmatched revascularization segments. Segments with matched no revascularization (no stress PET reversible defect and no revascularization) had unchanged MBF between the 2 PET scans. Thus, the authors show that if targeted revascularization is done in segments where PET reversible perfusion defects are noted, then one can expect to see an increase in post-PCI sMBF pertaining to those areas. They conclude that their study is the first study to evaluate quantitative MBF parameters by rubidium-82 PET MPI in patients with CAD pre and post intervention and could potentially serve as an attractive option to triage which patients and which territories would benefit from revascularization.

This finding of reduced SMBF in areas of stress perfusion defects showing improvement post-PCI could have implications when planning revascularization strategies. Trials, such as COURAGE, have failed to show improvement of routine anatomy-based revascularization compared to optimal medical therapy ${ }^{20}$ although sub-studies of COURAGE have highlighted that relief of residual ischemic burden on optimal medical therapy with PCI may have improved outcomes. ${ }^{26}$ These findings are now further solidified with data from the FAME and DEFER studies that have emphasized ischemia-guided revascularization. The current study conceptually fits into this paradigm showing that when using PET to identify ischemic areas, which are then specifically targeted for revascularization, there will likely be concomitant improvement in sMBF in those areas. Whether this translates to improved outcomes using the strategy employed in this study cannot be clarified based on this study.

While these data add to the prior studies on this topic, there are some significant limitations of this study: (a) small cohort of study subjects $(\mathrm{n}=19)$; (b) not all of the patients who had the initial PET underwent the second PET study, so there was inherent bias in patient selection of repeat PET which was driven by clinical symptoms; and (c) 5 of the 19 patients had either a ventricular paced rhythm or underlying left bundle branch block, and the influence of a paced ventricular rhythm on sMBF and CFR assessment is not fully understood. The authors acknowledge some of these limitations but argue that their primary focus of the study was to assess the impact of regional revascularization on regional MBF based upon PET perfusion imaging. Also one cannot conclusively deduce from their study the true impact of this change with revascularization given a very small patient population.

How do findings of Bober et al impact clinical practice? Exploring this question is central to help us understand the role of PET MBF assessment in the CAD diagnostic paradigm. There have been remarkable advances in pharmacologic therapies in the past two decades, and we know from studies such as COURAGE that even in patients with significant CAD, medical therapy has an important role to play. Prior to the advent of statins, almost all of these patients would be treated by revascularization, but that is certainly not the case anymore. Patients with small ischemic burden in the absence of other high-risk features on the stress MPI (SPECT or PET) seem to do better with initial trial of medical therapy when compared to revascularization. Testing the sMBF hypothesis of the current study in a patient group who do not undergo revascularization but do undergo aggressive medical therapies will help us better understand the role of PET MBF assessment in this cohort. Of note, the patients in the Bober et al study were on excellent medical therapy; however, the numbers are too small in the four groups to reach any conclusions. Future studies will also need to examine if improving regional MBF, as shown by PET, translates to better clinical outcomes. Thus, just focusing on regional segment flow improvement is just not sufficient justification for intervention rather what it would do for outcomes is most paramount.

In conclusion evidence mounts that revascularization of ischemic zones identified by clinically indicated stress PET in patients despite adequate medical therapy is associated with improved regional blood flow as demonstrated by PET. Larger studies performed in a prospective fashion using PET MPI focusing on hard outcomes and examining the role of sMBF in guiding revascularization are eagerly awaited and will establish the superiority of PET perfusion imaging guiding revascularization in clinical practice. 


\section{Funding}

None.

\section{Disclosures}

Dr. Ananthasubramaniam receives research grants from GE Healthcare and Astellas Pharma US Inc. Dr. Srivastava has no relevant disclosures.

\section{References}

1. Marwick TH, Shan K, Patel S, Go RT, Lauer MS. Incremental value of rubidium- 82 positron emission tomography for prognostic assessment of known or suspected coronary artery disease. Am J Cardiol 1997;80:865-70.

2. Yoshinaga K, Chow BJ, Williams K, Chen L, deKemp RA, Gar$\operatorname{rard} \mathrm{L}$, et al What is the prognostic value of myocardial perfusion imaging using rubidium-82 positron emission tomography? J Am Coll Cardiol 2006;48:1029-39.

3. Merhige M, Garza D, Sease D, Rowe RW, Tewson T, Emran A, et al Quantitation of the critically ischemic zone at risk during acute coronary occlusion using PET. J Nucl Med 1991;32:1581-6.

4. Gould KL, Johnson NP, Bateman TM, Beanlands RS, Bengel FM, Bober R, et al Anatomic versus physiologic assessment of coronary artery disease. Role of coronary flow reserve, fractional flow reserve, and positron emission tomography imaging in revascularization decision-making. J Am Coll Cardiol 2013;62:1639-53.

5. Hachamovitch R, Hayes SW, Friedman JD, Cohen I, Berman DS. Comparison of the short-term survival benefit associated with revascularization compared with medical therapy in patients with no prior coronary artery disease undergoing stress myocardial perfusion single photon emission computed tomography. Circulation 2003;107:2900-7.

6. Klocke FJ, Baird MG, Lorell BH, Bateman TM, Messer JV, Berman DS, et al ACC/AHA/ASNC guidelines for the clinical use of cardiac radionuclide imaging-executive summary: A report of the American College of Cardiology/American Heart Association Task Force on Practice Guidelines (ACC/AHA/ASNC Committee to Revise the 1995 Guidelines for the Clinical Use of Cardiac Radionuclide Imaging). J Am Coll Cardiol 2003;42:1318-33.

7. Dorbala S, Vangala D, Sampson U, Limaye A, Kwong R, Di Carli MF. Value of vasodilator left ventricular ejection fraction reserve in evaluating the magnitude of myocardium at risk and the extent of angiographic coronary artery disease: A $82 \mathrm{Rb}$ PET/CT study. J Nucl Med 2007;48:349-58.

8. El Fakhri G, Kardan A, Sitek A, Dorbala S, Abi-Hatem N, Lahoud $\mathrm{Y}$, et al Reproducibility and accuracy of quantitative myocardial blood flow assessment with (82)Rb PET: Comparison with (13)Nammonia PET. J Nucl Med 2009;50:1062-71.

9. Murthy VL, Naya M, Foster CR, Hainer J, Gaber M, Di Carli G, et al Improved cardiac risk assessment with noninvasive measures of coronary flow reserve. Circulation 2011;124:2215-24.

10. Ziadi MC, Dekemp RA, Williams KA, Guo A, Chow BJ, Renaud $\mathrm{JM}$, et al Impaired myocardial flow reserve on rubidium-82 positron emission tomography imaging predicts adverse outcomes in patients assessed for myocardial ischemia. J Am Coll Cardiol 2011;58:740-8.

11. Fukushima K, Javadi MS, Higuchi T, Lautamaki R, Merrill J, Nekolla SG, et al Prediction of short-term cardiovascular events using quantification of global myocardial flow reserve in patients referred for clinical 82Rb PET perfusion imaging. J Nucl Med 2011;52:726-32.

12. Rischpler C, Higuchi T, Fukushima K, Javadi MS, Merrill J, Nekolla SG, et al Transient ischemic dilation ratio in $82 \mathrm{Rb}$ PET myocardial perfusion imaging: Normal values and significance as a diagnostic and prognostic marker. J Nucl Med. 2012;53:723-30.

13. Bober RM, Thompson CD, Morin DP. The effect of coronary revascularization on regional myocardial blood flow as assessed by stress positron emission tomography. J Nucl Cardiol. 2016. doi: 10.1007/s12350-016-0442-2.

14. Naya M, Murthy VL, Taqueti VR, Foster CR, Klein J, Garber M, et al Preserved coronary flow reserve effectively excludes highrisk coronary artery disease on angiography. J Nucl Med 2014;55:248-55.

15. Sdringola S, Nakagawa K, Nakagawa Y, Yusuf SW, Boccalandro F, Mullani N, et al Combined intense lifestyle and pharmacologic lipid treatment further reduce coronary events and myocardial perfusion abnormalities compared with usual-care cholesterollowering drugs in coronary artery disease. J Am Coll Cardiol 2003;41:263-72.

16. Sdringola S, Gould KL, Zamarka LG, McLain R, Garner J. A 6 month randomized, double blind, placebo controlled, multi-center trial of high dose atorvastatin on myocardial perfusion abnormalities by positron emission tomography in coronary artery disease. Am Heart J 2008;155:245-53.

17. Patel MR, Bailey SR, Bonow RO, Chambers CE, Chan PS, Dehmer GJ, et al ACCF/SCAI/AATS/AHA/ASE/ASNC/HFSA/ HRS/SCCM/SCCT/SCMR/STS 2012 appropriate use criteria for diagnostic catheterization: A report of the American College of Cardiology Foundation Appropriate Use Criteria Task Force, Society for Cardiovascular Angiography and Interventions, American Association for Thoracic Surgery, American Heart Association, American Society of Echocardiography, American Society of Nuclear Cardiology, Heart Failure Society of America, Heart Rhythm Society, Society of Critical Care Medicine, Society of Cardiovascular Computed Tomography, Society for Cardiovascular Magnetic Resonance, and Society of Thoracic Surgeons. J Am Coll Cardiol 2012;59:1995-2027.

18. Hachamovitch R, Rozanski A, Shaw LJ, Stone GW, Thomson LE, Friedman JD, et al Impact of ischaemia and scar on the therapeutic benefit derived from myocardial revascularization vs medical therapy among patients undergoing stress-rest myocardial perfusion scintigraphy. Eur Heart J 2011;32:1012-24.

19. Patel MR, Dehmer GJ, Hirshfeld JW, Smith PK, Spertus JA. ACCF/SCAI/STS/AATS/AHA/ASNC/HFSA/SCCT 2012 Appropriate use criteria for coronary revascularization focused update: A report of the American College of Cardiology Foundation Appropriate Use Criteria Task Force, Society for Cardiovascular Angiography and Interventions, Society of Thoracic Surgeons, American Association for Thoracic Surgery, American Heart Association, American Society of Nuclear Cardiology, and the Society of Cardiovascular Computed Tomography. J Am Coll Cardiol 2012;59:857-81.

20. Boden WE, O'Rourke RA, Teo KK, Hartigan PM, Maron DJ, Kostuk WJ, et al Optimal medical therapy with or without PCI for stable coronary disease. N Engl J Med 2007;356:1503-16.

21. De Bruyne B, Fearon WF, Pijls NH, Barbato E, Tonino P, Piroth $\mathrm{Z}$, et al Fractional flow reserve-guided PCI for stable coronary artery disease. N Engl J Med 2014;371:1208-17.

22. Johnson NP, Gould KL. Integrating noninvasive absolute flow, coronary flow reserve, and ischemic thresholds into a comprehensive map of physiological severity. JACC Cardiovasc Imaging 2012;5:430-40. 
23. Johnson NP, Kirkeeide RL, Gould KL. Is discordance of coronary flow reserve and fractional flow reserve due to methodology or clinically relevant coronary pathophysiology? JACC Cardiovasc Imaging 2012;5:193-202.

24. Schelbert HR. Anatomy and physiology of coronary blood flow. J Nucl Cardiol 2010;17:545-54.

25. Danad I, Uusitalo V, Kero T, Saraste A, Raijmakers PG, Lammertsma AA, et al Quantitative assessment of myocardial perfusion in the detection of significant coronary artery disease:
Cutoff values and diagnostic accuracy of quantitative [(15)O]H2O PET imaging. J Am Coll Cardiol 2014;64: 1464-75.

26. Shaw LJ, Berman DS, Maron DJ, Mancini GB, Hayes SW, Hartigan PM, et al Optimal medical therapy with or without percutaneous coronary intervention to reduce ischemic burden: Results from the clinical outcomes utilizing revascularization and aggressive drug evaluation (COURAGE) trial nuclear substudy. Circulation 2008;117:1283-91. 